\title{
NEW NUMERICAL MATRIX METHODS OF SOLVING THE QUASI-ONE-DIMENSIONAL EFFECTIVE-MASS EQUATION
}

\author{
W. Salejda*, M.H. Tyc, J. Andrzejewski, M. Kubisa, J. Misiewicz, \\ M. JUST AND K. RYCZKo \\ Institute of Physics, Wrocław University of Technology \\ Wybrzeże Wyspiańskiego 27, 50-370 Wrocław, Poland
}

(Received January 14, 1999; in final form March 29, 1999)

\begin{abstract}
New efficient numerical methods of computing eigenvalues and eigenvectors of cuasi-one-dimensional effective-mass Hamiltonian with arbitrary coordinate dependence of charge carrier mass are presented. Within the proposed approach the effective-mass equation is replaced by a nonsymmetric or symmetric matrix eigenproblem which can be analysed numerically with the help of existing computer routines. The presented methods are verified in special semiconductor heterostructure cases that are solvable within other approaches. A generalization of the presented methods for nonparabolic materials is also discussed.
\end{abstract}

PACS numbers: 02.70.Bf, 73.20.Dx

\section{Introduction}

Physical properties of low-dimensional semiconductor heterostructures (e.g. quantum wells, quantum wires, and superlattices) are commonly interpreted theoretically within the effective-mass theory [1-11]. In this context the main task is aimed towards an accurate solution of one-dimensional effective-mass equation (1DEME) for envelope function (Eq. (3) in the next section) with the charge carrier mass $m$ being a function of $z$ coordinate (the $O Z$ axis is typically assumed parallel to the growth direction of the heterostructure).

If $m(z)=$ const, then 1DEME is equivalent to one-dimensional Schrödinger equation (1DSE) which, in general, camnot be solved analytically. Therefore various numerical methods have been proposed and applied [12-33]. Among them, the most popular are shooting methods [12-16] and global matrix methods [17-28].

*Electronic address: ssalejda@rainbow.if.pwr.wroc.pl 
The latter methods, based on the finite-difference approach, replaces 1DSE with a matrix eigenvalue problem. The matrix methods have the following advantages over shooting methods: (1) one needs no initial guess for eigenvalues and eigenvectors to start computation; (2) neither iteration nor relaxation procedure is employed; (3) one can use the most efficient and advanced computer algorithms worked out in a numerical linear algebra [24, 29-33].

The one-dimensional effective-mass eigenproblem is solved usually by means of transfer matrix formalism [3,28], shooting methods or variational approach [2]. These methods are less universal and general than global matrix methods mentioned in the case of 1DSE.

In this paper new numerical methods of solving 1DEME with an arbitrary (also continuous) dependence of charge carrier effective mass $m$ on the $z$ coordinate are presented. They are, to the authors' knowledge, the first extension of known global matrix methods to the 1DEME case. The proposed numerical approach can be applied to compute eigenenergies and wave functions of bound states in heterostructures with composition gradient, like ones regarded in [34].

Section 2 briefly presents physical foundations of our approach to the problem. Section 3 describes known matrix method for 1DSE with $m$ independent of $z$. The generalization of this method to the case $m=m(z)$ is presented in Sec. 4 . Section 5 discusses a way of including nonparabolicity effects in non-homogeneous materials into the proposed approach. Section 6 contains the results of computational tests, followed by our conclusions.

\section{The effective-mass equation}

Let us consider a crystal with parabolic electron (or hole) dispersion law $[35,36]$. The dependence of the kinetic energy $\mathcal{E}$ on the wave vector $k$ is, in a gencral case, anisotropic

$$
\mathcal{E}(k)=\frac{\hbar^{2}}{2} k \cdot \mathrm{m}^{-1} \cdot k=\frac{\hbar^{2}}{2} \sum_{i, j}\left(\mathrm{~m}^{-1}\right)_{i, j} k_{i} k_{j},
$$

where $m$ is the effective mass tensor. Here we will consider the case when in each heterostructure layer it has the form

$$
\mathrm{m}=\operatorname{diag}\left(m_{x x}, m_{y y}, m_{z z}=m\right),
$$

e.g., when $O Z$ is parallel to the [100] direction in III-V compounds with zinc-blende structure.

In the effective-mass approximation the envelope function $\varphi$ satisfies the Schrödinger equation

$$
[\mathcal{E}(-\mathrm{i} \nabla)+\mathcal{U}(r)] \varphi(r)=\varepsilon \varphi(r),
$$

where $\mathcal{E}(-\mathrm{i} \nabla)$ is the operator formed by replacing $k$ with $-\mathrm{i} \nabla$ in (1). The same procedure is applicable to the non-stationary case that is not considered here. Factorizing $\varphi(r)=\exp \left(k_{x} x+k_{y} y\right) \psi(z)$ and using Eqs. (1) and (2) one can write effective-mass equation for layered heterostructures as follows:

$$
\left[-\frac{\hbar^{2}}{2} \frac{\mathrm{d}}{\mathrm{d} z} \frac{1}{m(z)} \frac{\mathrm{d}}{\mathrm{d} z}+\mathcal{U}(z)\right] \psi(z)=\varepsilon \psi(z) .
$$


The kinetic energy of the transversal motion

$$
\varepsilon_{\perp}(z)=\frac{\hbar^{2}}{2}\left[\frac{k_{x}^{2}}{m_{x x}(z)}+\frac{k_{y}^{2}}{m_{y y}(z)}\right]
$$

may be considered as a part of the "effective potential" $\mathcal{U}$.

Let us note that more complicated and higher-order forms of the kinetic part of the effective-mass Hamiltonian can be found in [7, 9]; they are not considered here.

In the case when the effective mass is constant within specified intervals (e.g. when two layers made of $\mathrm{A}$ and $\mathrm{B}$ materials meet at the plane $z_{0}$ ):

$$
m(z)= \begin{cases}m_{\mathrm{A}} & \text { for } z<z_{0}, \\ m_{\mathrm{B}} & \text { for } z>z_{0},\end{cases}
$$

one can solve "ordinary" Schrödinger equations with constant masses $m_{i}$ $(i=\mathrm{A}, \mathrm{B})$ :

$$
\left[-\frac{\hbar^{2}}{2 m_{i}} \frac{\mathrm{d}^{2}}{\mathrm{~d} z^{2}}+\mathcal{U}(z)\right] \psi_{i}(z)=\varepsilon \psi_{i}(z),
$$

and match its solutions taking into account continuity conditions leading to conservation of the probability current

$$
j=\frac{\hbar}{2 \mathrm{i} m}\left(\psi^{*} \frac{\partial \psi}{\partial z}-\psi \frac{\partial \psi^{*}}{\partial z}\right) .
$$

The connection rules for $\psi$ and $\mathrm{d} \psi / \mathrm{d} z$ are cliscussed, e.g., in $[4-6,8]-$ here we consider their simplest form [1]:

$$
\psi_{\mathrm{A}}\left(z_{0}-\right)=\psi_{\mathrm{B}}\left(z_{0}+\right),\left.\quad \frac{1}{m_{\mathrm{A}}} \frac{\mathrm{d} \psi_{\mathrm{A}}}{\mathrm{d} z}\right|_{z_{0-}}=\left.\frac{1}{m_{\mathrm{B}}} \frac{\mathrm{d} \psi_{\mathrm{B}}}{\mathrm{d} z}\right|_{z_{0}+} .
$$

This is so-called direct matching procedure. It is the transfer matrix method foundation.

One should notice that the continuity condition is really satisfied only for full electron wave function (the product of the envelope function $\psi$ and the Bloch function $u k$ ). It means that Eqs. (4) are approximate only.

\section{Matrix methods of solving one-dimensional Schrödinger equation}

For the sake of clarity, let us first consider the simplest matrix method of solving 1DSE [25]. More sophisticated ones can be found, e.g., in [19, 22].

We start with the dimensionless form of 1DSE

$$
\left[-\frac{1}{m^{\prime}} \frac{\mathrm{d}^{2}}{\mathrm{~d} z^{\prime 2}}+G_{0} \mathcal{U}^{\prime}\left(z^{\prime}\right)\right] \psi^{\prime}\left(z^{\prime}\right)=G_{0} \varepsilon^{\prime} \psi^{\prime}\left(z^{\prime}\right)
$$

where

$$
m^{\prime}=\frac{m}{m_{0}}, \quad z^{\prime}=\frac{z}{l_{0}}, \quad \varepsilon^{\prime}=\frac{\varepsilon}{W_{0}}, \quad \mathcal{U}^{\prime}=\frac{\mathcal{U}}{W_{0}},
$$

$\psi^{\prime}\left(z^{\prime}\right)=\sqrt{l_{0}} \psi\left(l_{0} z^{\prime}\right)$ denotes the dimensionless wave function, $m_{0}, l_{0}$ and $W_{0}$ are the characteristic mass, length and energy, respectively, and $G_{0}=2 m_{0} l_{0}^{2} W_{0} / \hbar^{2}$. In particular, for $m_{0}=m_{e}, l_{0}=1 \AA$ and $W_{0}=1 \mathrm{eV}$ we get $G_{0}=0.262468291$. 
In the following we will omit primes (') for simplicity.

Solving (5) by the matrix methods consists of discretization of the equation and reducing it to an algebraic eigenproblem. Equation (5) is discretized over grid of points in a finite interval $\langle a, b\rangle$ :

$$
z_{i}=a+i s \quad \text { for } i=0,1, \ldots, N+1,
$$

where

$$
s=\frac{b-a}{N+1} \text {. }
$$

The wave function is represented by the vector

$$
\boldsymbol{\Psi}=\left[\Psi_{1}, \Psi_{2}, \ldots, \Psi_{N}\right]^{\mathrm{T}}=\left[\psi\left(z_{1}\right), \psi\left(z_{2}\right), \ldots, \psi\left(z_{N}\right)\right]^{\mathrm{T}} .
$$

The potential is also defined on the grid points: $\mathcal{U}_{i}=\mathcal{U}\left(z_{i}\right)$.

We impose the following boundary conditions for the envelope function:

$\psi(a)=\psi(b)=0 \quad \Rightarrow \quad \Psi_{0}=\Psi_{N+1}=0$.

Before the presentation of further computational details, let us discuss the meaning of boundary conditions (6). They correspond to infinite (non-penetrable) potential barriers placed at the ends of the interval. For well-like potentials (with finite number of local minima and $\lim _{z \rightarrow+\infty} \mathcal{U}(z)=\mathcal{U}_{+}, \lim _{z \rightarrow-\infty} \mathcal{U}(z)=\mathcal{U}_{-}$), envelope functions of bound states (if they exist) satisfy boundary conditions

$$
\psi(-\infty)=\psi(+\infty)=0,
$$

which can be well approximated by (6) when the points $a, b$ are far enough from the envelope function localization area (where $|\psi|$ is relatively large).

In this approximation, extended states (if any) disappear due to.infinite barriers and they camnot be found. Instead, "artificial" higher bound states arise.

The differential operator appearing in (5) can be approximated by finite differences $[19-22,25]$.

Let us apply in (5) the standard three-point formula for the second derivative

$$
\left.\frac{\mathrm{d}^{2} \psi}{\mathrm{d} z^{2}}\right|_{z_{i}}=\frac{\Psi_{i-1}-2 \Psi_{i}+\Psi_{i+1}}{s^{2}}+\mathrm{O}\left(s^{2}\right),
$$

where $\mathrm{O}\left(s^{2}\right)$ denotes the approximation error. Taking $m_{0}=m$, we get a set of $N$ equations at the points $z_{i}(i=1,2, \ldots, N)$ :

$$
-\Psi_{i-1}+\left(2+\tilde{\mathcal{U}}_{i}\right) \Psi_{i}-\Psi_{i+1}=\widetilde{\varepsilon} \Psi_{i}
$$

with the reduced potential and energy clefined as

$$
\tilde{\mathcal{U}}_{i}=G_{0} s^{2} \mathcal{U}_{i}, \quad \widetilde{\varepsilon}=G_{0} s^{2} \varepsilon .
$$

Rewriting (7) in the matrix form and taking boundary conditions (6) into account, we get the matrix eigenproblem

$H \boldsymbol{\Psi}=\widetilde{\varepsilon} \boldsymbol{\Psi}$,

with the symmetric tridiagonal matrix (STM) $N \times N$

$$
\mathrm{H}=\left[\begin{array}{cccccc}
a_{1} & b_{2} & 0 & 0 & \cdots & 0 \\
b_{2} & a_{2} & b_{3} & 0 & \cdots & 0 \\
0 & b_{3} & a_{3} & b_{4} & \cdots & 0 \\
\vdots & & \ddots & \ddots & \ddots & \vdots \\
0 & \cdots & 0 & b_{N-1} & a_{N-1} & b_{N} \\
0 & \cdots & 0 & 0 & b_{N} & a_{N}
\end{array}\right]=\operatorname{trid}\left(b_{i}, a_{i}, b_{i+1}\right)
$$


where

$$
a_{i}=2+\tilde{U}_{i}, \quad b_{i}=-1 .
$$

It is known that any STM with non-zero off-diagonal elements has $N$ different eigenvalues [31], and it is also known that eigenvalues of one-dimensional Hamiltonian corresponding to the bound states are nondegenerate [37]. The lowest eigenvalues of $\mathrm{H}$ correspond to energies of lowest bound states (cf. (8)). One has to reject numerically obtained "artificial" eigenenergies $\varepsilon_{i} \geq \min (\mathcal{U}(a), \mathcal{U}(b))$, because they result from the boundary conditions (infinite barriers at $z=a, b$ ). For some potentials (e.g. a shallow half-infinite well) one has to reject all obtained solutions - it means there is no "real" bound states.

Because we are not interested in all eigenvalues of $H$ and it would be too time-consuming to evaluate all of them (usually $N=10^{3}$ to $10^{5}$ ), it is convenient to employ a bisection method. It allows us to look only for eigenvalues lying in a specified interval, and it is very accurate and numerically stable. It is based on the following theorem [38]:

The number of eigenvalues of STM (9) less than $x$ is equal to the number of negative elements in the sequence

$$
u_{1}=a_{1}-x, \quad u_{i}=a_{i}-x-\frac{b_{i}^{2}}{u_{i-1}} \quad \text { for } i=2, \ldots, N .
$$

More sophisticated methods of finding eigenvalues of tridiagonal matrices based on bisection and Sturm sequences are presented in [39]; dependence of the eigenenergy numerical errors on the number of grid points is also investigated there.

After evaluating the eigenvalues $\widetilde{\varepsilon}$ of the matrix $H$ one can find its eigenvectors $\boldsymbol{\Psi}$. It can be done in different ways.

A simple and efficient method of computing eigenvectors of STM (9) (or any block-tridiagonal matrix) is $D W S Z^{\dagger} \operatorname{method}$ [40-42]. Unlike the standard $\mathrm{QR}^{\ddagger}$ algorithm [32,33], based on iterative orthogonal similarity transformations, it allows $u=10$ calculate one selected eigenvector and does not require computer memory space for any $N \times N$ matrix.

We define recursively two auxiliary sequences

$$
\begin{aligned}
& \Delta_{1}^{-}=\left(a_{1}-\widetilde{\varepsilon}\right)^{-1}, \\
& \Delta_{i}^{-}=\left(a_{i}-\widetilde{\varepsilon}-b_{i}^{2} \Delta_{i-1}^{-}\right)^{-1} \text { for } i=2,3, \ldots, N-1, \\
& \Delta_{N}^{+}=\left(a_{N}-\widetilde{\varepsilon}\right)^{-1}, \\
& \Delta_{i}^{+}=\left(a_{i}-\widetilde{\varepsilon}-b_{i+1}^{2} \Delta_{i+1}^{+}\right)^{-1} \text { for } i=N-1, N-2, \ldots, 2,
\end{aligned}
$$

by means of which the components $\Psi_{i}$ can be calculated using the formula

$$
\Psi_{i}= \begin{cases}-\Delta_{i}^{-} b_{i+1} \Psi_{i+1} & \text { for } i=k-1, k-2, \ldots, 1 \\ -\Delta_{i}^{+} b_{i} \Psi_{i-1} . & \text { for } i=k+1, k+2, \ldots, N\end{cases}
$$

'This abbreviation comes from the authors' names: $\mathrm{Dy}$, Wu, Spratlin and Zheng.

$t_{\mathrm{Q}}$ denotes an orthogonal matrix, $\mathrm{R}-$ a right-triangle matrix. 
We put $\Psi_{k}=1$, for the chosen value of index $k$, and calculate the other components $\Psi_{i}$ using (11). Next we normalize the obtained eigenvector: $\widetilde{\boldsymbol{\Psi}}=\boldsymbol{\Psi} /\|\boldsymbol{\Psi}\|$.

In order to verify the accuracy of numerical results one can use the Schrödinger equation at $z_{k}$ point $(H \tilde{\Psi})_{k}=\widetilde{\varepsilon} \widetilde{\Psi}_{k}$, the explicit form of which reads as

$$
b_{k} \widetilde{\Psi}_{k-1}+\left(a_{k}-\tilde{\varepsilon}\right) \widetilde{\Psi}_{k}+b_{k+1} \widetilde{\Psi}_{k+1}=0 .
$$

Due to a numerical error of the eigenvalue $\widetilde{\varepsilon}$, the above equation is not fulfilled. In computations we replace it with the inequality

$$
\left|b_{k} \widetilde{\Psi}_{k-1}+\left(a_{k}-\widetilde{\varepsilon}\right) \widetilde{\Psi}_{k}+b_{k+1} \widetilde{\Psi}_{k+1}\right|<r
$$

for some specified accuracy $r$. If it is not satisfied, one should choose another starting point $k$. The best choice is the point at $\left|\widetilde{\Psi}_{k}\right|$ maximum.

It can be difficult to indicate the proper starting point. The practice shows that it is efficient to find sucl an index $k$ for which the quantity

$$
\left|b_{k} \Psi_{k-1}+\left(a_{k}-\tilde{\varepsilon}\right) \Psi_{k}+b_{k+1} \Psi_{k+1}\right|
$$

reaches its minimum [39] or, which is equivalent and computationally more convenient,

$$
\left|b_{k}^{2} \Delta_{k-1}^{-}+\left(a_{k}-\tilde{\varepsilon}\right)+b_{k+1}^{2} \Delta_{k+1}^{+}\right|
$$

does. In this way we can find the starting point already during computing the sequences $\left\{\Delta_{\dot{i}}^{ \pm}\right\}$and the components of $\Psi$ are calculated only once.

However, there are some potentials for which this method may fail. The double quantum well with wide and/or high barrier is an example. In this case eigenvalues are very close to each other; the wave functions values are so small in the barrier that numerical precision is lost and computed eigenvectors are invalid.

The other method of computing eigenvectors is the inverse iteration method $[22,29,30,43]$. It also fails in the case of almost-degenerate eigenvalues.

\section{Application of matrix methods to effective-mass equation}

Let us write the effective-mass equation (3) in the dimensionless form analogous to (5):

$$
\left[-\frac{\mathrm{d}}{\mathrm{d} z} w(z) \frac{\mathrm{d}}{\mathrm{d} z}+G_{0} \mathcal{U}(z)\right] \psi(z)=G_{0} \varepsilon \psi(z) .
$$

From here on we will denote by $w$ (for convenience) the reciprocal of the effective mass $\left(m^{-1}\right)$.

In order to transform (12) into a matrix eigenproblem we approximate the differential operator $\frac{\mathrm{d}}{\mathrm{d} z} w \frac{\mathrm{d}}{\mathrm{d} z}$ by finite differences. It can be done in different ways; beloil' we present two simplest ones.

\subsection{Single grid method (SGM)}

We transform the first term appearing in (12) as follows:

$$
D(z)=\left.\frac{\mathrm{d}}{\mathrm{d} z} w \frac{\mathrm{d} \psi}{\mathrm{d} z}\right|_{z}=\left.\left.\frac{\mathrm{d} w}{\mathrm{~d} z}\right|_{z} \frac{\mathrm{d} \psi}{\mathrm{d} z}\right|_{z}+\left.w(z) \frac{\mathrm{d}^{2} \psi}{\mathrm{d} z^{2}}\right|_{z},
$$

then we use standard three-point formulae for the second and first derivatives. We get 


$$
\begin{gathered}
D\left(z_{i}\right)=\frac{1}{s^{2}}\left[\left(w_{i}+\frac{w_{i-1}-w_{i+1}}{4}\right) \Psi_{i-1}-2 w_{i} \Psi_{i}\right. \\
\left.+\left(w_{i}+\frac{w_{i+1}-w_{i-1}}{4}\right) \Psi_{i+1}\right]+\mathrm{O}\left(s^{2}\right) .
\end{gathered}
$$

Introducing the quantities $\widetilde{\varepsilon}$ and $\tilde{\mathcal{U}}_{i}$, according to (8), we get the set of $N$ equations for the points $z_{i}(i=1,2, \ldots, N)$. Imposing boundary conditions (6), we obtain matrix eigenproblem $\mathbf{H} \boldsymbol{\Psi}=\widetilde{\boldsymbol{\varepsilon}} \boldsymbol{\Psi}$ with

where

$$
\mathrm{H}=\operatorname{trid}\left(b_{i}, a_{i}, c_{i}\right) \text {, }
$$

$$
a_{i}=2 w_{i}+\tilde{\mathcal{U}}_{n}, \quad b_{i}=-w_{i}+\frac{w_{i+1}-w_{i-1}}{4}, \quad c_{i}=-w_{i}-\frac{w_{i+1}-w_{i-1}}{4} .
$$

Matrix (13) is tridiagonal, but nonsymmetric. Nonsymmetric matrices are generally more difficult to diagonalize than symmetric ones. However, in this case, if not too rigorous assumptions about the function $w(z)$ and the distance $s$ between grid points, namely

$$
\left|w_{i-1}-w_{i+1}\right|<4 w_{i}
$$

are fulfilled, the inequality $b_{i+1} c_{i}>0$ is satisfied and $\mathrm{H}$ is a quasisymmetric tridiagonal matrix [30]. Such a matrix has the spectrum identical to one with the same diagonal elements $a_{i}$ and off-diagonal elements $b_{i}^{\prime}=\sqrt{b_{i} c_{i}}$. Transition between the quasisymmetric matrix $\mathrm{H}$ and the symmetric matrix $\mathrm{H}^{\prime}$ can be done with the similarity transformation

$$
\Delta^{-1} H \Delta=H^{\prime}
$$

where $\Delta$ is a diagonal matrix with diagonal elements

$$
\Delta_{1}=1, \quad \Delta_{i}=\left(\frac{\prod_{j=2}^{i} b_{i}}{\prod_{j=1}^{i-1} c_{i}}\right)^{1 / 2} \quad \text { for } i=2, \ldots, N .
$$

In this way one can use the Dean method to find eigenvalues of $\mathrm{H}$. Transformation (14) need not be physically performed; it is equivalent to a slight modification of the sequence $\left\{u_{i}\right\}$ in comparison with (10):

$$
u_{1}=a_{1}-x, \quad u_{i}=a_{i}-x-\frac{b_{i} c_{i-1}}{u_{i-1}} \quad \text { for } i=2,3, \ldots, N .
$$

The eigenvectors of $\mathrm{H}^{\prime}$ can be computed with DWSZ method. Multiplying them by $\Delta$ one gets eigenvectors of $H$. One can also apply DWSZ method indirectly to the matrix $H(13)$, but in that case the sequences $\left\{\Delta_{i}^{ \pm}\right\}$have to be redefined

$$
\begin{aligned}
& \Delta_{1}^{-}=\left(a_{1}-\tilde{\varepsilon}\right)^{-1}, \\
& \Delta_{i}^{-}=\left(a_{i}-\widetilde{\varepsilon}+b_{i} c_{i-1} \Delta_{i-1}^{-}\right)^{-1} \text { for } i=2,3, \ldots, N-1, \\
& \Delta_{N}^{+}=\left(a_{N}-\widetilde{\varepsilon}\right)^{-1}, \\
& \Delta_{i}^{+}=\left(a_{i}-\widetilde{\varepsilon}+b_{i+1} c_{i} \Delta_{i+1}^{+}\right)^{-1} \text { for } i=N-1, N-2, \ldots, 2 .
\end{aligned}
$$

The formulae for $\Psi_{i}$ read then

$$
\Psi_{i}= \begin{cases}-\Delta_{i}^{-} c_{i} \Psi_{i+1} & \text { for } i=k-1, k-2, \ldots, 1 \\ -\Delta_{i}^{+} b_{i} \Psi_{i-1} & \text { for } i=k+1, k+2, \ldots, N\end{cases}
$$




\subsection{Single grid method in the case of ideal abrupt heterojunction}

Let us consider a jump of the effective mass at the point $z_{j}$. Here we propose a method of symmetrizing the matrix (13).

Let us assume that the function $w(z)$ and its derivative are given by

$$
w\left(z_{i}\right)=\left\{\left.\begin{array}{ll}
w_{\mathrm{A}} & \text { for } i<j, \\
\mu & \text { for } i=j, \\
w_{\mathrm{B}}, & \text { for } i>j,
\end{array} \quad \quad \mathrm{\textrm {d } w}\right|_{z_{i}} \quad= \begin{cases}0 & \text { for } i \neq j, \\
g / s & \text { for } i=j,\end{cases}\right.
$$

where the magnitudes of $g$ and $\mu$ are unknown. We have to find them to get a symmetric matrix eigenproblem. In order to do this, we write the Schrödinger equation for the points $j-1, j$ and $j+1$ and apply three-point formulae for the first and the second derivatives. After some algebraic transformations we find that the following relations should be fulfilled:

$$
+\frac{g}{2 s}-\frac{\mu}{s^{2}}=-\frac{w_{j-1}}{s^{2}}, \quad-\frac{w_{j+1}}{s^{2}}=-\frac{g}{2 s}-\frac{\mu}{s^{2}} .
$$

Solving the above set of equations we get

$$
\mu=\frac{w_{j-1}+w_{j+1}}{2}, \quad g=w_{j+1}-w_{j-1} \text {. }
$$

Finally we obtain the eigenproblem for symmetric tridiagonal matrix of the form (9), where

and

$$
b_{i}= \begin{cases}-w_{\mathrm{A}} & \text { for } i \leq j \\ -w_{\mathrm{B}} & \text { for } i>j\end{cases}
$$

$$
a_{i}= \begin{cases}2 w_{\mathrm{A}}+G_{0} s^{2} \mathcal{U}_{i} & \text { for } i<j \\ w_{\mathrm{A}}+w_{\mathrm{B}}+G_{0} s^{2} \mathcal{U}_{j} & \text { for } i=j \\ 2 w_{\mathrm{B}}+G_{0} s^{2} \mathcal{U}_{i} & \text { for } i>j\end{cases}
$$

\subsection{Double grid method (DGM)}

In this method we define the second (auxiliary) grid of points, shifted by $s / 2$ relative to the main grid

$$
z_{i+1 / 2}=a+(i+1 / 2) s \text {. }
$$

Let us treat the product $w \frac{\mathrm{d} \psi}{\mathrm{d} z}$ appearing in (12) as a single function $\varphi$; we have now

$$
-\frac{\mathrm{d}}{\mathrm{d} z} \varphi(z)-G_{0} \mathcal{U}(z) \psi(z)=G_{0} \varepsilon \psi(z)
$$

We approximate values of $\varphi$ at points $z_{i \pm 1 / 2}$ using the three-point formula. We get

$$
\Phi_{i \pm 1 / 2}= \pm w_{i \pm 1 / 2} \frac{\Psi_{i \pm 1}-\Psi_{i}}{s}+\mathrm{O}\left(s^{2}\right)
$$

Now let us apply three-point formula to the function $\varphi$ in Eq. (15):

$$
\begin{aligned}
\left.\frac{\mathrm{d} \varphi}{\mathrm{d} z}\right|_{z_{i}} & =\frac{\Phi_{i+1 / 2}-\Phi_{i-1 / 2}}{s}+\mathrm{O}\left(s^{2}\right) \\
& =\frac{1}{s^{2}}\left[w_{i-1 / 2} \Psi_{i-1}-\left(w_{i-1 / 2}+w_{i+1 / 2}\right) \Psi_{i}+w_{i+1 / 2} \Psi_{i+1}\right]+\mathrm{O}\left(s^{2}\right) .
\end{aligned}
$$


Therefore the values of effective mass are defined on the auxiliary grid $z_{i+1 / 2}$ and the values of wave function $\psi$ on the main grid with integer indices, as in SGM.

Continuity of the function $\varphi=\frac{1}{n} \frac{\mathrm{d} \psi}{\mathrm{d} z}$ is required for continuity of the probability current (cf. (4)). Multiplying the discretized version of (15) by $s$ and passing with $s$ to 0 we get

$$
\lim _{s \rightarrow 0}\left[\varphi\left(z-\frac{s}{2}\right)-\varphi\left(z+\frac{s}{2}\right)\right]=\lim _{s \rightarrow 0}\left\{G_{0} s[\varepsilon-\mathcal{U}(z)] \psi(z)\right\}=0,
$$

so conditions (4) are fulfilled.

Applying formula (16) we can write the set of $N$ equations for points $z_{i}$ $(i=1,2, \ldots, N)$ in the form $\mathrm{H} \boldsymbol{\Psi}=\widetilde{\varepsilon} \boldsymbol{\Psi}$, where $\mathrm{H}$ is STM (9) with

$$
a_{i}=w_{i-1 / 2}+\tilde{\mathcal{U}}_{i}+w_{i+1 / 2}, \quad b_{i}=-w_{i-1 / 2} .
$$

It is easy to see that for step-like function $m(z)$ one gets a matrix identical to one defined in subsection $4.2-$ in this case both methods are equivalent.

Eigenvalues of $\mathrm{H}$ can be found by means of the Dean method, and eigenvectors with DWSZ method.

\section{Including nomparabolicity effects}

The parabolic conduction band approximation that we have used so far is in many cases insufficient [1]. The simplest way to go beyond its limitations is to consider the effective mass as energy-dependent: $m=m(\varepsilon)$ and $w=w(\varepsilon)$ [10]. It requires to compute the elements of the tridiagonal matrix $H$ before each evaluation of the $\left\{u_{i}\right\}$ sequence for different values of $\widetilde{\varepsilon}$ (or during this process) and significantly slows down calculations. However, if the interval which is searched for an eigenvalue is not too wide, one can linearize the dependence $w(\widetilde{\varepsilon})$ introducing position-dependent coefficients $\alpha$ and $\beta$ :

$$
w(\widetilde{\varepsilon} ; z)=\alpha(z)+\beta(z) \widetilde{\varepsilon} .
$$

Defining $\alpha$ and $\beta$ on the grid, we get new expressions for the elements of sequences $\left\{u_{i}\right\}$ (cf. (10)), e.g., for DGM - substituting

$$
\begin{aligned}
& a_{i}=\alpha_{i-1 / 2}+\alpha_{i+1 / 2}+\left(\beta_{i-1 / 2}+\beta_{i+1 / 2}\right) \widetilde{\varepsilon}+\widetilde{\mathcal{U}}_{i}, \\
& b_{i}=-\alpha_{i-1 / 2}-\beta_{i-1 / 2} \widetilde{\varepsilon} .
\end{aligned}
$$

In this way numerical calculations can be significantly simplified.

\section{Tests and comparison of the methods}

We have tested both methods (SGM and DGM) in three cases which are solvable analytically or by other numerical methods that give accurate results. We have also used them recently for calculations in [44].

\subsection{The Legendre equation}

One-dimensional effective-mass equation is a special case of self-adjoint form of a second-order ordinary differential equation

$$
\left\{\frac{\mathrm{d}}{\mathrm{d} z}\left[p(z) \frac{\mathrm{d}}{\mathrm{d} z}\right]+q(z)\right\} \psi(z)=0 .
$$


The self-adjoint form of the Legendre equation reads as

$$
\left\{\frac{\mathrm{d}}{\mathrm{d} z}\left[\left(1-z^{2}\right) \frac{\mathrm{d}}{\mathrm{d} z}\right]+l(l+1)\right\} P_{l}(z)=0
$$

and its solutions are the Legendre polynomials $P_{l}(z)$ for $l=0,1,2, \ldots$ (they are considered within the interval $\langle-1,1\rangle)$. If we choose the interval $\langle a, b\rangle$ ends so that $P_{l}(a)=P_{l}(b)=0$, then $P_{l}$ should be one of the eigenfunctions $\psi$ and $l(l+1)$ should be one of the eigenvalues $\varepsilon$ of one-dimensional effective-mass equation (3) with $m(z)=\frac{1}{1-z^{2}}$ and $\mathcal{U}(z)=0=$ const.

Table I presents exact eigenvalues $\varepsilon^{(l)}$ and the eigenvalues $\varepsilon^{(l, 2)}$ and $\varepsilon^{(l, 1)}$ computed with DGM and SGM, respectively. The equation was solved within intervals

$$
\left\langle a^{(l)}, b^{(l)}\right\rangle=\left\langle\left|z_{0}^{(l)}\right|,-\left|z_{0}^{(l)}\right|\right\rangle \in\langle-1,1\rangle
$$

where $\pm z_{0}^{(l)}$ are the $l$-th Legendre polynomial zeros furthest from the point $z=0$. We used $N=2^{10}-1=1023$ and $2^{12}-1=4095$ grid points. In the bisection method the eigenvalue accuracy was set to $10^{-10}$. Additionally, for $N=1023$ we computed the eigenvectors with the DWSZ method and compared them with the Legendre polynomials, calculating

$$
\left\|d^{(l)}\right\|=\left\|\boldsymbol{\Psi}^{(l)}-\boldsymbol{P}_{l}\right\|=\sum_{i=1}^{N}\left[\psi_{i}^{(l)}-P_{l}\left(z_{i}\right)\right]^{2} .
$$

All the calculations in this example and following ones were performed on a $\mathrm{PC}$ with extended variables (64-bit mantissa).

TABLE I

Analytical and numerical results for Legendre polynomials.

\begin{tabular}{r|r|r|r|r|r|r|r}
\hline \hline$l$ & \multirow{2}{*}{$\varepsilon^{(l)}$} & \multicolumn{2}{|c|}{$N=4095$} & \multicolumn{4}{|c}{$N=1023$} \\
\cline { 3 - 8 } & & \multicolumn{1}{|c|}{$\varepsilon^{(l, 2)}$} & \multicolumn{1}{|c}{$\varepsilon^{(l, 1)}$} & \multicolumn{1}{c}{$\varepsilon^{(l, 2)}$} & \multicolumn{1}{c}{$\varepsilon^{(l, 1)}$} & $\left\|d^{(l, 2)}\right\|$ & $\left\|d^{(l, 1)}\right\|$ \\
\hline 2 & 6 & 5.9999998 & 6.0000000 & 5.9999976 & 6.0000000 & $1.86 \cdot 10^{-13}$ & $1.29 \cdot 10^{-21}$ \\
3 & 12 & 11.9999985 & 11.9999992 & 11.9999766 & 11.9999866 & $4.76 \cdot 10^{-11}$ & $1.56 \cdot 10^{-11}$ \\
4 & 20 & 19.9999939 & 19.9999954 & 19.9999017 & 19.9999257 & $1.31 \cdot 10^{-9}$ & $9.55 \cdot 10^{-10}$ \\
5 & 30 & 29.9999820 & 29.9999849 & 29.9997119 & 29.9997579 & $1.11 \cdot 10^{-8}$ & $8.80 \cdot 10^{-9}$
\end{tabular}

The numbers in Table I point to slightly better consistence with analytical results in the case of the single grid method.

\subsection{Finite rectangular quantum well}

The second example we have used for testing our methods was $200 \AA$ wide and $266 \mathrm{eV}$ deep quantum well in the conduction band of $\mathrm{GaAs} / \mathrm{Al}_{0.3} \mathrm{Ga}_{0.7} \mathrm{As}$ structure, which is shown in Fig. 1.

The effective masses are: $m_{\mathrm{A}}=0.067 m_{e}, m_{\mathrm{B}}=0.085 m_{\mathrm{e}}$.

Energy levels $\varepsilon_{n}^{(\mathrm{dm})}$ were calculated by direct matching of wave functions in the well $\left(\psi_{\mathrm{A}}(z)=\cos (k z)\right.$ or $\left.\psi_{\mathrm{A}}(z)=\sin (k z)\right)$ and in the barrier $\left(\psi_{\mathrm{B}}(z)=\mathrm{e}^{-q|z|}\right)$ 


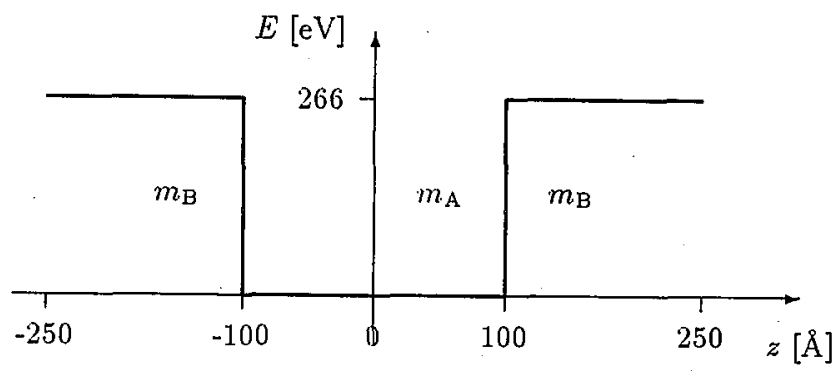

Fig. 1. Quantium well test potential.

using conditions (4). It required to find numerically solutions of a transcendental equation.

The energies $\varepsilon_{n}^{(2)}$ and $\varepsilon_{n}^{(1)}$ were calculated with DGM and SGM, respectively. The grid of $N=4999$ points was put on the interval $\langle-250 \AA,+250 \AA)$. The eigenvalue accuracy in the bisection procedure was set to $10^{-10} \mathrm{eV}$. We put the mean value of $\mathcal{U}$, i.e. $133 \mathrm{eV}$, in the points of potential jump (they were grid points). We treated similarly the jumps of $w(z)$ in SGM.

The eigenvectors calculated with the DWSZ method were tested by a comparison with the eigenfunctions $\psi^{(\mathrm{dm})}$ resulting from the direct matching procedure. Similar to the previous example, we computed $\left\|d_{l}\right\|=\left\|\boldsymbol{\Psi}_{l}-\boldsymbol{\Psi}^{(\mathrm{dm})}\right\|$.

\section{TABLE II}

Numerical results for quantum well potential.

\begin{tabular}{c|r|r|r|c|c}
\hline \hline$n$ & $\varepsilon_{n}^{(\mathrm{dm})}[\mathrm{eV}]$ & $\varepsilon_{n}^{(2)}[\mathrm{eV}]$ & $\varepsilon_{n}^{(1)}[\mathrm{eV}]$ & $\left\|d_{n}^{(2)}\right\|$ & $\left\|d_{n}^{(1)}\right\|$ \\
\hline 1 & 10.33454 & 10.33456 & 10.33340 & $1.44 \times 10^{-11}$ & $2.32 \times 10^{-8}$ \\
2 & 41.21578 & 41.21584 & 41.21170 & $1.50 \times 10^{-10}$ & $5.60 \times 10^{-8}$ \\
3 & 92.15064 & 92.15066 & 92.14326 & $4.18 \times 10^{-9}$ & $7.90 \times 10^{-8}$ \\
4 & 161.66611 & 161.66597 & 161.65783 & $5.77 \times 10^{-7}$ & $6.26 \times 10^{-7}$ \\
5 & 243.65630 & 243.68756 & 243.68694 & $3.43 \times 10^{-3}$ & $3.43 \times 10^{-3}$
\end{tabular}

The results are shown in Table II. It can be seen that the double grid method gives results closer to "real" ones obtained by direct matching.

For both matrix methods, the decrease in accuracy can be seen for higher energy states. It results from a deeper penetration of their wave functions into the barriers, so the boundary conditions (6) are less precise (e.g. for the state $n=5$ the value $\psi(z= \pm 250 \AA)$ is $10^{4}$ times more than for $\left.n=1\right)$. The accuracy for higher states can be improved by the use of wider interval $\langle a, b\rangle$.

\subsection{Double quantum well}

As the next test we have used more complicated structure - a double quantum well made from the same materials as before (Fig. 2). 


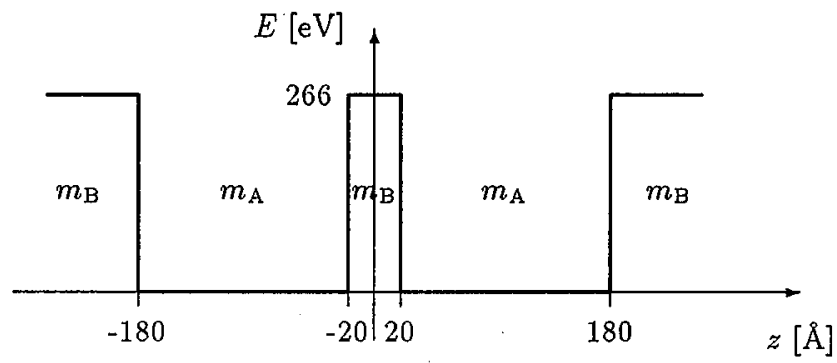

Fig. 2. Double quantum well test potential.

We again compared the results with those obtained with direct matching. In both matrix methods we worked on the interval $\langle-400 \AA,+400 \AA\rangle$ and the grid of $N=7999$ points. The accuracy of eigenenergies search was $10^{-10} \mathrm{eV}$.

\section{- TABLE III}

Numerical results for donble quantum well potential.

\begin{tabular}{c|r|r|r}
\hline \hline$n$ & $\varepsilon_{n}^{(\mathrm{dm})}[\mathrm{eV}]$ & $\varepsilon_{n}^{(2)}[\mathrm{eV}]$ & $\varepsilon_{n}^{(1)}[\mathrm{eV}]$ \\
\hline 1 & 14.80001 & 14.80004 & 14.79806 \\
2 & 15.29230 & 15.29233 & 15.29032 \\
3 & 58.75170 & 58.75177 & 58.74532 \\
4 & 60.97611 & 60.97619 & 60.96930 \\
5 & 129.98035 & 129.98032 & 129.98153 \\
6 & 136.12710 & 136.12708 & 136.11590 \\
7 & 222.63822 & 222.63784 & 222.63762 \\
8 & 236.37426 & 236.37405 & 236.36639
\end{tabular}

The results are shown in Table III. They can be interpreted in the same way as for a single well.

Because the results of DGM and SGM differ from each other, we have investigated how the $\mathcal{U}$ and $w$ jump at the boundaries of the structure layers influences this difference. Instead of step-like functions $\mathcal{U}(z)$ and $w(z)$ we used, partially following [10], continuous functions of Fermi distribution type

$$
f(T, \mu ; \varepsilon)=\left[\exp \left(\frac{\varepsilon-\mu}{T}\right)+1\right]^{-1} ;
$$

naturally $T, \mu$ and $\varepsilon$ are in length units.

We performed the calculations for $N=3999$ grid points, using the potential and the effective mass given below

$$
\mathcal{U}(z)=266(1-f(T, 80 ;|| x|-100|)),
$$




$$
m(z)=[0.085-0.018 f(T, 80 ;|| x|-100|)] m_{e} ;
$$

all lengths are expressed in $\AA$, all energies in eV. We have put $3.0 \AA$ and $0.1 \AA$ as the broadening parameter $T$.

\section{TABLE IV}

Numerical results for smoothed double quantum well potential.

\begin{tabular}{c|r|r|r|r}
\hline \hline $\mathrm{n}$ & \multicolumn{2}{|c|}{$T=3.0 \AA$} & \multicolumn{2}{|c}{$T=0.1 \AA$} \\
\cline { 2 - 5 } & $\varepsilon_{n}^{(2)}[\mathrm{eV}]$ & $\varepsilon_{n}^{(1)}[\mathrm{eV}]$ & $\varepsilon_{n}^{(2)}[\mathrm{eV}]$ & $\varepsilon_{n}^{(1)}[\mathrm{eV}]$ \\
\hline 1 & 15.39673 & 15.39675 & 14.80073 & 14.80111 \\
4 & 63.01743 & 63.01756 & 60.97852 & 60.97953 \\
7 & 223.98683 & 223.98731 & 222.63921 & 222.64788
\end{tabular}

Selected results are presented in Table IV. It can be clearly seen that for greater $T$ (smoother functions $\mathcal{U}(z)$ and $m(z)$ ) the differences between the results of the two methods are smaller. Therefore, taking into account the data from Table III we can state that DGM works well in wider class of cases.

\subsection{Eigenstates in Fibonacci-type superlattices}

In Ref. [44] we calculated, within the trace maps formalism [28, 45], reviewed recently in [46], the spectra of the Landauer conductance of semiconductor generalized Fibonacci-type superlattices, which is related to the electron tunneling coefficient: $\sigma_{\mathrm{L}} \sim T / R=T /(1-T)$. The $l$-th order structure $\mathcal{S}_{l}$ is a quasiperiodical chain of wells $\mathcal{A}$ (e.g. GaAs) and barriers $\mathcal{B}$ (e.g. $\mathrm{Al}_{x} \mathrm{Ga}_{1-x} \mathrm{As}$ ), arranged with respect to the following rules:

$$
\mathcal{S}_{1}=\mathcal{B}, \quad \mathcal{S}_{2}=\mathcal{A}, \quad \mathcal{S}_{l+1}=\left(\mathcal{S}_{l}\right)^{n}\left(\mathcal{S}_{l-1}\right)^{m},
$$

where the exponents $m, n \geq 1$ mean repetition of the components.

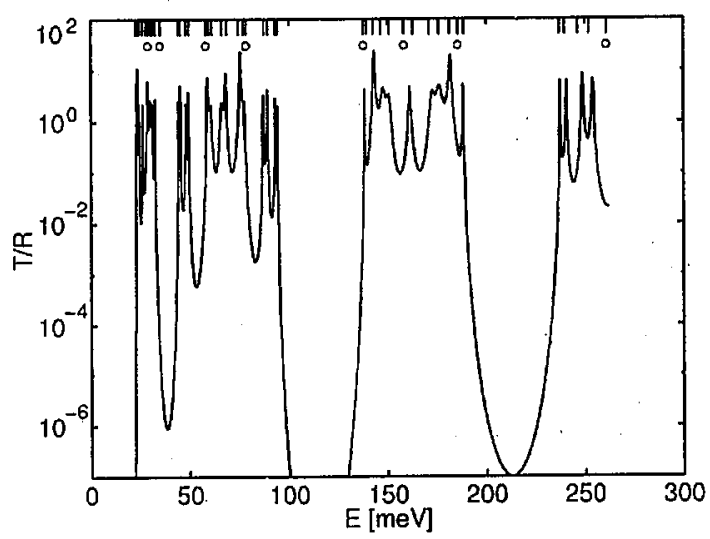

Fig. 3. The Landauer conductance spectrum and computed eigenenergies (short vertical lines at the top) for $\mathrm{GaAs} / \mathrm{Al}_{0.3} \mathrm{Ga}_{0.7} \mathrm{As}$ Fibonacci-type superlattice $(l=7$, $n=2, m=1)$. Extra eigenenergies are marked with "o". 
We used DGM to compute the eigenenergies of these structures, putting infinite potential barriers next to the external $\mathcal{B}$ components. As is shown in Fig. 3 , the results agree with the maxima of $T / R$ obtained within the traic maps formalism with free boundary conditions. The extra eigenenergies are the consequence of the applied boundary conditions.

\section{Conclusions}

Known numerical matrix methods of solving quasi-one-dimensional Schrödinger equation [25] are extended to the case of one-dimensional effective-mass equation in this paper. Two different discretization schemes of $1 \mathrm{DEME}$ are considered. They result in replacing effective-mass equation with a nonsymmetric or symmetric matrix eigenproblem. They allow us to solve 1DEME (3) with an arbitrary dependence $m(z)$. It is also possible to include band nonparabolicity.

The performed test results show that our nonstandard methods are correct and efficient. They prove the superiority of the double grid method, which gives accurate results for the continuous as well as for the step-like dependence $m(z)$. The single grid method, in the latter case, is less accurate. The next advantage of the double grid method is that it uses a symmetric matrix, which is more convenient in numerical calculations and needs less computer memory for storage. In addition, the probability current in this method is conserved in an obvions way.

The methods worked out can be used to compute energy levels in semiconductor heterostructures. In addition they can be applied to second-order differential equations of which self-adjoint form is an eigenproblem with "zero" boundary conditions.

\section{References}

[1] G. Bastard, Wave Mechanics Applied to Semiconductor Heterostructures, Les Editions de Physique, Les Ulis Cedex 1988, Chapters I and III.

[2] C. Weisbuch, B. Vinter, Quantum: Semiconductor Structures: Fundamentals and Applications, Academic Press, San Diego 1991, Ch. II.

[3] K. Iguchi, Int. J. Mod. Phys. B 11, 2157 (1997).

[4] T. Ando, S. Mori, Surf. Sci. 113, 124 (1982).

[5] T. Ando, S. Wakahara, H. Akera, Phys. Rev. B 40, 11609 (1989).

[6] B. Laikhtman, Phys. Rev. B 46, 4769 (1992) and references [3-8] therein.

[7] B.A. Foreman, Phys. Rev. B 52, 12241 (1995).

[8] B.A. Foreman, Phys. Rev. Lett. 80, 3823 (1998).

[9] R. Balian, D. Bessis, Phys. Rev. B 51, 17624 (1995).

[10] J.H. Nelson, R.C. Miller, C.W. Tu, S.K. Sputz, Phys. Rev. B 36, 8063 (1987).

[11] M.G. Burt, J. Phys. Condens. Matter 4, 6651 (1992).

[12] J. Killingbeck, Microcomputer Algorithms, Hilger, Bristol 1991.

[13] J. Killingbeck, G. Jolicard, Phys. Lett. A 172, 313 (1993).

[14] F.Y. Hajj, J. Phys. B 13, 4521 (1980).

[15] L. Greengard, V. Rokhlin, Commun. Pure Appl. Math. XLIV, 419 (1991). 
[16] W.H. Press, B.P. Flannery, S.A. Teukolsky, W.T. Vetterling, Numerical Recipes. Art of Scientific Computing, Cambridge University Press, Cambridge 1992, Ch. 16.

[17] D.G. Truhlar, J. Comput. Phys. 10, 123 (1972).

[18] P.J. Cooney, E.P. Kantor, Z. Vager, Am. J. Phys. 49, 76 (1987).

[19] R. Guardiola, J. Ros, J. Comput. Phys: 45, 374 (1982).

[20] V. Fack, G. Vanden Berghe, J. Phys. A 18, 3355 (1985).

[21] V. Fack, G. Vanden Berghe, Comput. Phys. Commun. 39, 187 (1986).

[22] B. Lindberg, J. Chem. Phys. 88, 3805 (1988).

[23] G.C. Groenenboom, H.M. Buck, J. Chem. Phys. 92, 4374 (1990).

[24] In Ref. [16], Ch. 11.

[25] J. Van der Maelen Uría, S. García-Granda, A. Menéndez-Velázquez, Am. J. Phys. 64, 327 (1996).

[26] R. Vargas, J. Garza, A. Vela, Phys. Rev. B 53, 1954 (1996) and references [18-23] therein.

[27] M. Znojil, Phys. Lett. A 223, 411 (1996).

[28] W. Salejda, P. Szyszuk, Physica A 252, 547 (1998).

[29] Handbook for Automatic Computations, Vol. 2, Linear Algebra, Eds. J.H. Wilkinson, C. Reinsch, Springer-Verlag, Heidelberg 1971.

[30] J.H. Wilkinson, The Algebraic Eigenvalue Problem, Clarendon Press, Oxford 1965, Ch. 7 .

[31] J. Stoer, R. Bulirsch, Introduction to Numerical Analysis, Springer-Verlag, New York 1983, Chapters 5 and 6.

[32] E. Anderson, Z. Bai, C. Bischof, J. Demme!. J. Dongarra, J. Du Croz, A. Greenbaum, S. Hammerling, A. McIKenney, S. Ostrouchov, D. Sorensen, LAPACK User's Guide - Release 2.0, SIAM, Philadelphia 1995.

[33] B.T. Smith, Matrix Eigensystems Routines - EISPACK Guide, Lecture Notes in Computer Science, Vol. 6, Springer-Verlag, Berlin 1976; B.W. Garbow, J.M. Boyle, J.J. Dongarra, C.B. Moler, Matrix Eigensystem Routines - EISPACK Guide Extension, Lecture Notes in Computer Science, Vol. 61, Springer-Verlag, Berlin 1977.

[34] L. Leibler, Phys. Rev. B 11, 3037 (1975).

[35] E. Madelung, Introduction to Solid-State Theory, Springer-Verlag, Berlin 1978, Ch. 2.

[36] W.A. Harrison, Solid State Theory, McGraw-Hill, New York 1970, Ch. 2.

[37] L.D. Landau, E.M. Lifshitz, Quantum Mechanics. Nonrelativistic Theory, OGIZ, Moscow 1948 (in Russian).

[38] P. Dean, Rev. Mod. Phys. 44, 127 (1972).

[39] M. Just, M.Sc. Thesis, Report SPR-333/1998, Institute of Pliysics, Wrocław University of Technology, Wrocław 1998; W. Salejda, M. Just, Numerical Methods of Solving the Schrödinger Equation. Program Package: MARRS, Proceedings of Conference on Computer Physics, 4.5, Institute of Nuclear Physics, Kraków, 1997.

[40] K.S. Dy, S.Y. Wu, T. Spratlin, Phys. Rev. B 20, 4237 (1979).

[41] Z. Zheng, J. Phys. Condens. Matter 19, L689 (1986).

[42] W. Salejda, Int. J. Mod. Phys. B 9, 1475 (1995). 
[43] A. Kiełbasiński, H. Schwetlick, Numeryczna algebra liniowa, WNT, Warszawa. 1992 (in Polish).

[44] W. Salejda, M. Kubisa, J. Misiewicz, K. Ryczko, M.H. Tyc, Acta Phys. Pol. A 94, 514 (1998).

[45] W. Salejda, Physica A 232, 769 (1996).

[46] Y. Avishai, D. Berend, V. Tkachenko, Int. J. Mod. Phys. B 11, 3525 (1997). 